reactive derivative synthesized. This substance, called fluorescamine, is itself barely fluorescent, reacts more or less instantaneously with amino groups to yield the fluorescent product, and can therefore be used as the basis of an assay (Böhlen et al., Arch. Biochem., 155, 213 ; 1973). The fluorescent response is linear with concentration, and the sensitivity is limited by the presence of traces of primary amines as impurities. Under these circumstances the method can be used to estimate $500 \mathrm{ng}$ and up of protein. If, however, impurities of low molecular weight are stripped out by gel filtration in an automated apparatus, this lower level falls by at least an order of magnitude. The response depends of course on the lysine content of the protein, so that for an accurate determination a calibration with the same protein is necessary, but this drawback is, as Böhlen et al. point out, no greater than that of the Folin reaction and other colorimetric methods.

In an accompanying report from the same laboratory, Stein et al. (ibid., 203) describe the most spectacular application of fluorescamine. If it is used in place of ninhydrin in an amino acid analyser, and the products are monitored by fluorescence instead of absorption, then the sensitivity begins to approach the picomolar range, that is to say $1 \mu \mathrm{g}$ of protein suffices for amino acid analyses of the highest quality. Hydrolysis is performed in the usual way, and derivatization of the amino acids follows their separation on the ion-exchange columns. Very narrow columns can then be used, with correspondingly greater flow rates, less diffusion, and thus better separation, for a given pumping speed. The reaction is practically instantaneous, and does not need heating.

The possibilities that the method now opens should exercise the minds of many biochemists. Stein et al. have already found it possible to determine free amino acids in microlitre volumes of plasma and to perform analyses on minute quantities of proteins in slices of stained poly. acrylamide gels, because the ammonia generated during the hydrolysis of the polyacrylamide does not interfere with the fluorescamine reaction, or itself give rise to a highly fluorescent product. A commercial version of the fluorescencebased amino acid analyser is in prospect.

\section{LIQUIDS}

\section{Centenary Remembered}

from a Correspondent

THE third of a series of conferences on the liquid state, started by the Institute of Physics and Physical Society in 1963, was held at the University of Kent from April 16 to 18 , and was on the work of van der Waals and the relationships of this work with contemporary and later developments.

Professor J. S. Rowlinson (Imperial College, London) opened the programme with a paper on the legacy of van der Waals which is to be published in Nature in due course. Professor Rowlinson gave a careful account of the history of van der Waals's work on the equation of state, on the critical region and on mixtures, and showed how far it amounted to a synthesis of already existing ideas without detracting in any way from its greatness. Several later speakers returned to this theme of "history repeats itself". For example, Drs A. J. B. Cruikshank and J. W. Goodwin (University of Bristol) showed how van der Waals's ideas are still helpful in interpreting residual functions of liquid mixtures, and Drs Y. de Ribaupierre and F. D. Manchester (University of Toronto) suggested that the hydrogen-palladium system can usefully be compared with a van der Waals gas, the distension of the palladium lattice acting rather like a long-range attraction between the hydrogens.

One of the principal themes was the interplay of different methods. For example Professor J. E. Enderby and his colleagues (University of Leicester) described a comparison of X-ray and neutron studies of strong aqueous solutions of $\mathrm{NiCl}_{2}$, the scattering power of the nickel for neutrons being changed by isotope substitution. Some very interesting conclusions were drawn about this complicated system. Also of interest is the fact that considerable information is obtainable from light scattering (Professor G. Benedek (MIT)) and ultrasonics (Professor D. Sette (University of Rome)) in spite of the fact that the wavelengths are much greater than atomic spacings.

It was also of interest to hear about the state of the art of computer simula- tion of liquid-like systems, for example, diatomic liquids, fused salts, hard nonspherical molecules, which could certainly not have been tackled until quite recently. Highlights were the invited paper by Dr A. Rahman (Argonne National Laboratory) showing what could be done with only thirty-two "molecules", and the film by Dr R. W. Hockney et al. (University of Reading) showing strange "collective" movements in an ionic liquid near its solidification point.

One of the growth points of theory is the study of models of polar liquids. Professor G. Rushbrooke (University of Newcastle upon Tyne) showed how perturbation theory, introducing dipolar interactions into a reference system (for example, the hard sphere fluid), can now be carried through systematically. Dr O. R. McDonald (Royal Holloway College, London) showed the good accuracy now obtainable. Agreement with Monte Carlo calculations is found to be very good except in the notorious region near the triple point which always proves difiicult to handle theoretically.

Perhaps the most interesting contribution was the last in the programme. Professor Benedek gave several fascinat. ing examples of the application of optical methods to biological problems. It is now possible, for example, to compare the overall size of a ribosome when it is resting and when it is in the process of synthesizing protein. Laser optics has now attained such refinement that the flow velocity of blood in retinal arteries (about $1 \mathrm{~cm} \mathrm{~s}^{-1}$ ) can now be measured by Doppler shift in the frequency of the scattered light.

This most enjoyable conference highlighted the advances that have been made since 1963. There is now a good understanding of "simple" liquids which provides a sound foundation for treating more complicated systems.

\title{
Radio Source Associated with OH Source ON-1
}

WINNBERG and colleagues are presently carrying out a search for compact radio sources close to class $1 \mathrm{OH}$ sources, using the Westerbork Synthesis Radio Telescope and the $100 \mathrm{~m}$ at Effelsberg. Their search has proved successful with the first object examined, and in next Monday's Nature Physical Science (June 4) they describe observations of a source near ON-1.

The radio source is at

$$
\begin{aligned}
& \alpha=20 \text { h } 08 \text { min } 09.8 \pm 0.1 \mathrm{~s} \\
& \delta=31^{\circ} 22^{\prime} 41^{\prime \prime} \pm 1^{\prime \prime}
\end{aligned}
$$

and the $\mathrm{OH}$ source position is

$$
\begin{aligned}
& \alpha=20 \text { h } 08 \text { min } 09.8 \pm 0.5 \mathrm{~s} \\
& \delta=31^{\circ} 22^{\prime} 41^{\prime \prime} \pm 15^{\prime \prime}
\end{aligned}
$$

which, as Winnberg et al. point out, seems to suggest that the quoted $\mathrm{OH}$ position (from studies at CalTech) is more accurate than the listed errors would imply.

The radio source was unresolved at $5 \mathrm{GHz}$ (WSRT observations) but revealed as a point source at $10.7 \mathrm{GHz}$ (Effelsberg data). Comparison of flux densities at the two frequencies suggests a thermal spectrum with considerable opacity even at the higher frequency. Although only an idealized model can be presented at this stage, the source may, it seems, represent the first stage in the evolution of an $\mathrm{H}$ II region.

Most class $1 \mathrm{OH}$ sources are found near large, evolved $\mathrm{H}$ II regions because that is where radio astronomers have searched. But it now seems that surveys should be made taking no account of the presence of continuum sources, and that the known sample may be heavily biased by this selection effect. 